\title{
Zum Massenhaushalt des Baby Glacier, Axel Heiberg Island, kanadische Hocharktis
}

Gletscher stehen in dauerndem Massen- und Energieaustausch mit der Umgebung. Die Massenänderungen eines Beobachtungsjahres werden durch die sogenannten Massenhaushaltserhebungen erfaßt, die die Grundlage für das Studium der Wechselbeziehungen zwischen Gletscher und Klima ergeben.

Seit 1959 werden durch die Axel Heiberg Island Expeditionen der McGill Universität in Montreal insbesondere auf dem White Glacier, einem subpolaren Talgletscher von $39 \mathrm{~km}^{2}$ Fläche mit wohldefiniertem Einzuggebiet, jährlich Massenhaushaltsmessungen durchgeführt (MÜLLER et al., 1961 und 1963, MÜLLER, 1962, ADAMS, 1966). Auf dem viel kleineren Baby Glacier $\left(0,6 \mathrm{~km}^{2}\right)$ wurden gleichzeitig Vergleichsmessungen aufgenommen. ADAMS (1966) hat die Daten der Haushaltsjahre 1959/60 bis 1961/62 ausgewertet. Nachstehend wird die Meßreihe 1959/60 bis 1975/76 des Baby Glacier gesamthaft dargestellt und diskutiert. Der Baby Glacier liegt südwestlich des White Glacier in einem kleinen nach SW geöffneten Tal an einem Bergzug, der im 1313 m hohen Black Crown Peak gipfelt. Die steile Zunge liegt bei $710 \mathrm{~m}$ ü. M., der höchste Punkt des Gletschers auf $1165 \mathrm{~m}$.

Wie auf dem White Glacier werden die Massenhaushaltsbestimmungen mit der sogenannten direkten glaziologischen Methode durchgeführt. An einzelnen Stellen auf dem Gletscher werden die Massenänderungen gegenüber einem Referenzhorizont im Schneeoder Eisprofil gemessen, unterhalb desselben idealerweise kein Massenverlust oder -gewinn mehr stattfindet.

Der Bezugshorizont wird durch Messungen an eingebohrten Stangen ermittelt. Durch Schneefall, gefrierendes Regenwasser oder windverfrachteten Schnee und durch Schmelzen von Schnee und Eis und Wegfließen des Wassers ändert sich die Masse häufig. Die Massenänderung zwischen zwei Begehungen wird als sogenannte Bilanz bestimmt. Die kontinuierliche Akkumulation oder Ablation ist aus praktischen Gründen kaum zu bestimmen, da beide Prozesse oft in zu rascher Folge auftreten, hingegen kann immer eine Massenbilanz erhalten werden.

Der Massengewinn von einem Tiefstand im Herbst bis zu einem Maximum nach dem Winter heißt Winterbilanz, $b_{w}$, der Verlust bis zum nächsten Tiefstand im Herbst, $b_{s}$, ist die Sommerbilanz, die Summe der beiden ist die Jahres- oder Nettobilanz, $b_{n}$.

$b_{w}+b_{s}=b_{n}$
Eine Schwierigkeit liegt in der Tatsache begründet, daß die Bilanzminima oben und unten auf dem Gletscher nicht gleichzeitig auftreten, jedoch ist dieser Zeitpunkt beim Baby Glacier recht scharf definiert, da dessen Höhenerstreckung klein ist. Die Zeitpunkte der Massentiefstände bestimmen das natürliche Haushaltsjahr, wobei aber auf anderen Gletschern gelegentlich mit festen Daten gearbeitet wird.

Die Bilanzmessungen auf dem Baby Glacier basieren auf einer sich stark ändernden Zahl von Meßstangen (2 bis 15) sowie einer unterschiedlichen Anzahl von Begehungen pro Jahr, was sich in der Genauigkeit der Ergebnisse äußert.

\section{Ergebnisse zum Massenhaushalt des Gletschers}

Das Geschehen auf dem Gletscher innerhalb eines Jahres kann anhand der Haushaltsjahre mit häufigen Begehungen abgeschätzt werden. Massenbilanzkurven (Fig. 1) steigen im Frühling und Herbst steil an und sind während der Wintermonate bemerkenswert flach. Dies erklärt sich durch die Niederschlagsverhältnisse; die arktischen Winter sind sehr trocken. Das Niederschlagsmaximum ist im Juli/August, doch überwiegt dann die Ablation, so daß die Bilanzkurven fallen. Die Muster der Bilanzwerte auf dem Gletscher sind recht veränderlich. Fig. 2 zeigt eine Isolinienkarte der spezifischen (auf die Einheitsfläche bezogenen) Nettobilanzen für das Haushaltsjahr 1969/70. Das «Ablationshoch» auf der Gletscherzunge entspricht den höheren Temperaturen in den tieferen Lagen, verstärkt durch intensivere Einstrahlung auf diesem gegen Südwesten geneigten Gletscherteil. In stark negativen Haushaltsjahren ist hier die spezifische Nettobilanz deutlich negativer als auf einem gleich hoch gelegenen, aber weniger nach Süden exponierten Gebiet des White Glacier.

Die spezifischen Nettobilanzen zeigen oben auf dem Gletscher einen Höchststand gegen die Talmitte hin. Dies ist nicht nur durch die Niederschlagsverteilung und Windverfrachtung, sondern vor allem durch die Bildung von sogenanntem "superimposed ice» zu erklären. Dieses Eis entsteht durch Wiedergefrieren von Schmelzwasser in noch winterkalten tieferen Schich-

Jürg Alean und Prof. Dr. Fritz Müller, Geographisches Institut ETHZ, Sonneggstraße 5, 8006 Zürich. 
Fig. 2 und 3: Legende schon in der Figur vorhanden. $\triangleright$

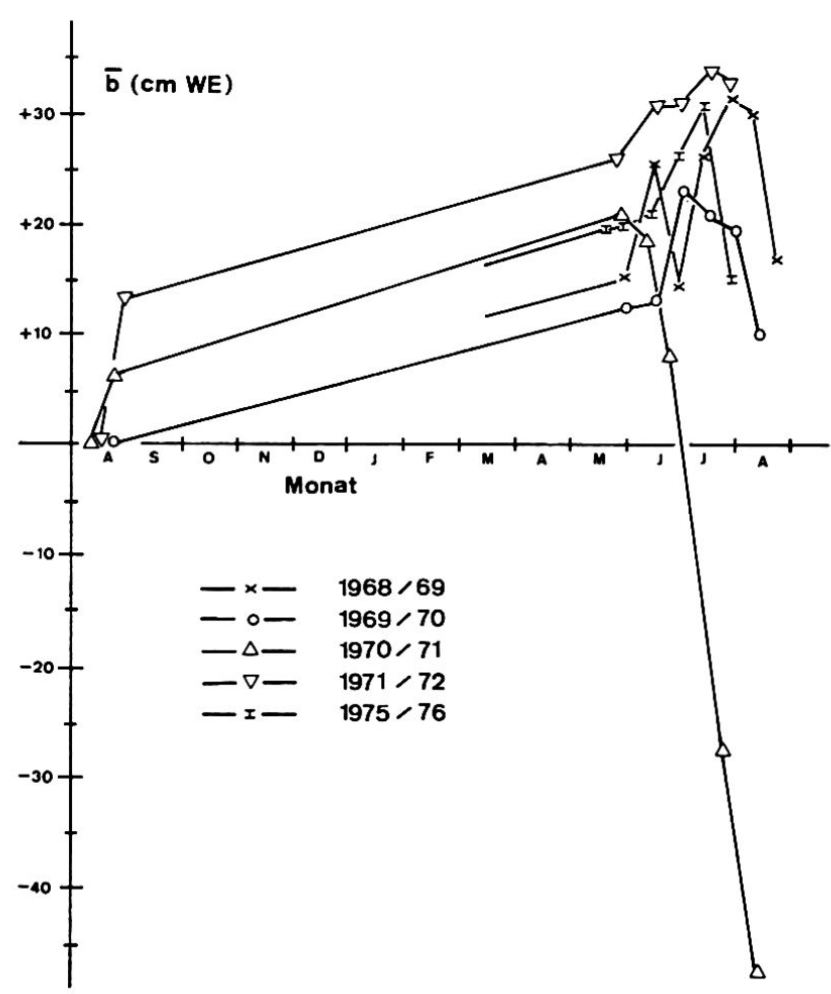

Fig. 1: Bilanzkurve für die Jahre mit mehreren Begehungen des Gletschers. Ausgangspunkt ist jeweils die minimale Masse des vorangehenden Jahres.

ten des Schneeprofils (MÜLLER, 1962; KOERNER, 1970). Normalerweise bildet es sich auf der Sommeroberfläche des vorhergehenden Haushaltsjahres. Es wächst dann nach Einsetzen der Schmelze von unten her der Schneeoberfläche entgegen und wird bei genügendem Abbau der Schneedecke selber wieder abgeschmolzen. Fig. 3 zeigt in Form einer Isolinienkarte für das gleiche Haushaltsjahr 1969/70 den Maximalstand an «superimposed ice», wie er etwa Mitte August erreicht wurde. Gezeigt wird zudem der Nettogewinn in Form dieses Eises am Ende des Haushaltsjahres. Offensichtlich wurde nur unten auf dem Gletscher solches Eis exponiert und abgeschmolzen. Das entsprechende $\mathrm{Zu}$ rückweichen der Isolinien nach oben wird durch Pfeile verdeutlicht. Das Maximum der Bildung von «superimposed ice» wird in der Talmitte beobachtet, weil sich dort Schmelzwasser von den Bergflanken sammelt. Die Wirkung auf das Muster der Nettobilanzen (Fig. 2) ist offensichtlich. Für 5 Haushaltsjahre konnte für den Baby Glacier der «superimposed ice»-Anteil an der
Nettobilanz bestimmt werden. Für Jahre mit positiver Bilanz erreicht er 1968/69 96\%, im Jahre 1971/72 16\%, im Mittel etwa 50\%. Da der Baby Glacier außerdem die Firnlinie meistens erreicht, ist er somit kaum zum Baffin-Typ (BAIRD, 1952) zu rechnen.

In Fig. 4 werden die wesentlichsten Bilanzgrößen veranschaulicht. 1972 bis 1974 mußte wegen einer fehlenden Begehung die Zweijahressumme angegeben werden. Die 5 Winterbilanzen, die erfaßt wurden, sind ungefähr gleich, während die Sommerbilanzen und somit auch die Nettobilanzen stark schwanken. Typisch sind viele leicht positive Nettobilanzen, deren Gewinn aber durch die wenigen, stark negativen $\mathrm{Bi}$ lanzen verloren geht. Die Verluste der Jahre 1959/60 und 1961/62 waren besonders groß. Diese Nettobilanzunterschiede sind das Ergebnis der relativ konstanten Winterniederschläge und der stärker schwankenden Sommertemperaturen, die die Sommerbilanz entsprechend stark verändern.

Ein zunächst vermuteter Trend $\mathrm{zu}$ immer weniger negativen Bilanzen scheint sich zu bestätigen. Der Durchschnitt der errechneten Nettobilanzen ist minus $6 \mathrm{~cm}$ Wasseräquivalent. Der Gletscher erfuhr also unter den klimatischen Bedingungen des Beobachtungszeitraumes einen nur geringen Massenschwund.

In gemäßigten Breiten ist die Lage der Gleichgewichtslinie (der Ort aller Punkte mit der Nettobilanz 0) ein wichtiger Parameter für den Massenhaushalt, da er ungefähr mit der Firnlinie identisch und deswegen leicht zu bestimmen ist. Wegen dem "superimposed ice» trifft dies für arktische Gletscher nicht zu. Für den Baby Glacier konnte zwar eine enge Korrelation zwischen der Höhe der Gleichgewichtslinie und der Nettobilanz berechnet werden, jedoch wird dies zu oft sinnlos, dann nämlich, wenn die Gleichgewichtslinie oberhalb oder unterhalb des Gletschers verläuft, was durchaus nicht selten ist. Der Baby Glacier ist dann gänzlich Ablations- oder Akkumulationsgebiet.

\section{Vergleich mit anderen Gletschern und mit Klimadaten}

Ein Vergleich der Massenbilanzen des Baby Glacier mit den bis anhin ausgewerteten des White Glacier ergibt bei einer guten Korrelation $(r=0.95$ bei $n=6)$, da $\beta$ die Nettobilanz des Baby Glacier immer fast genau das Doppelte derjenigen des White Glacier ist. 

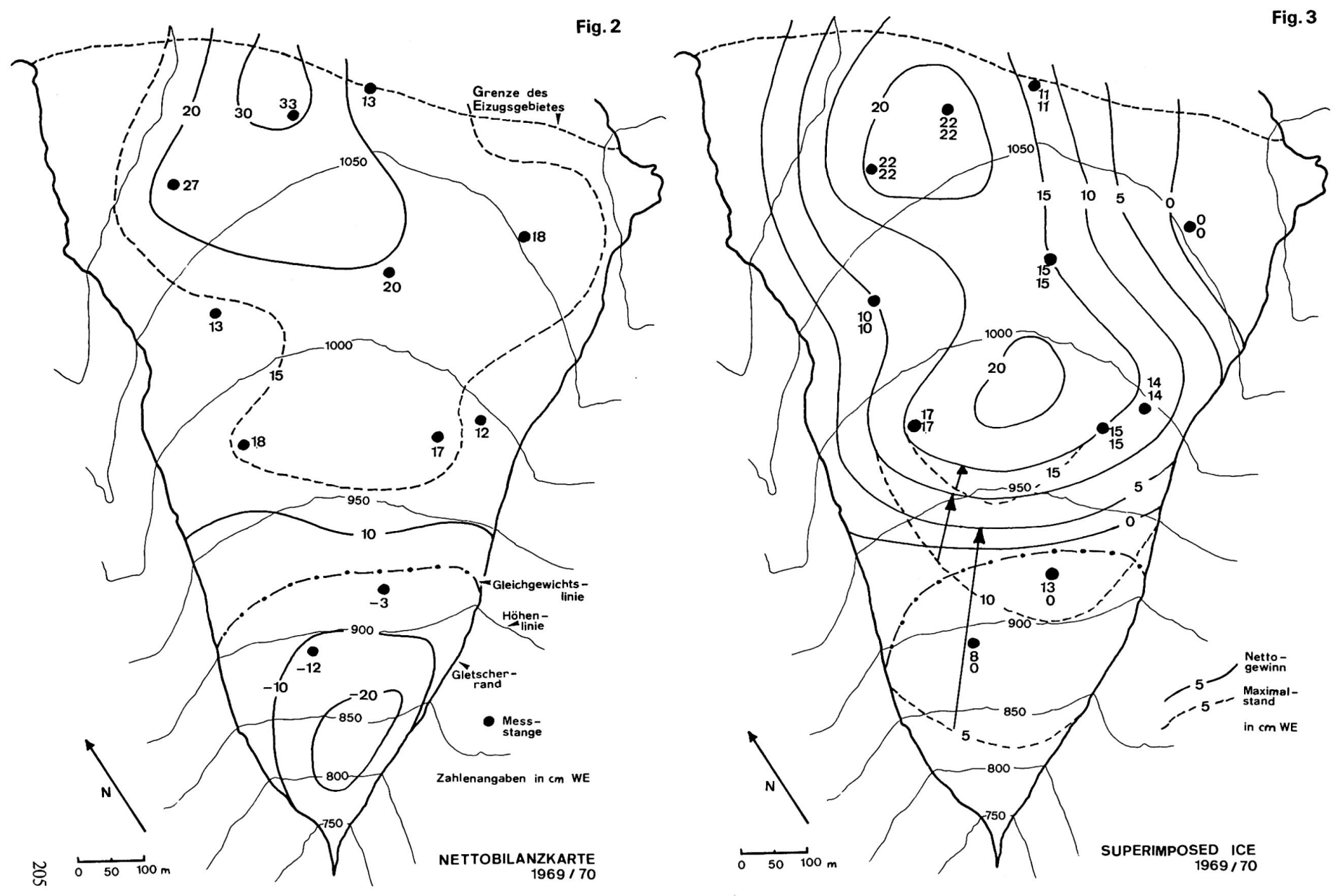


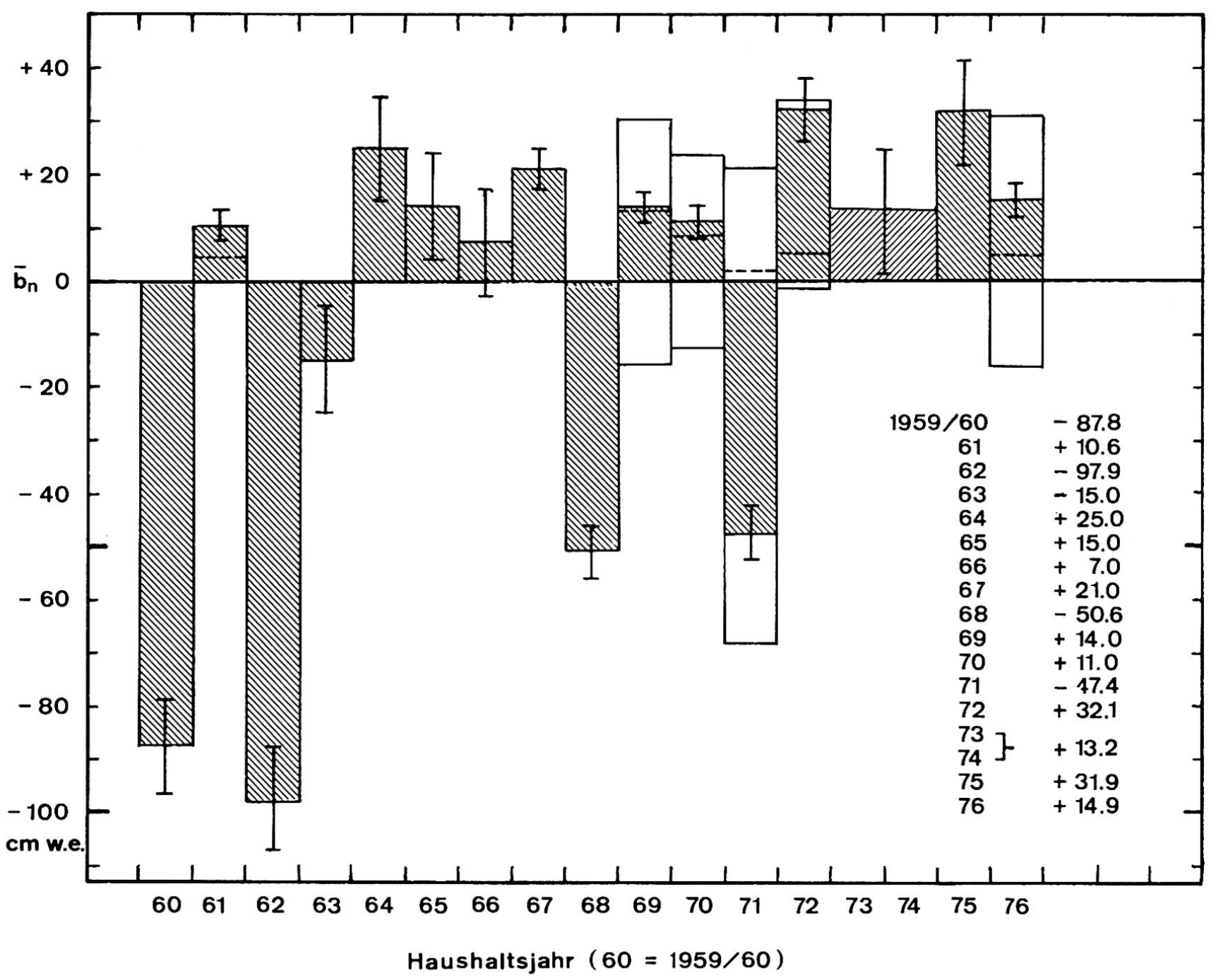

Fig. 4: Massenhaushalt Baby Glacier 1959-1976.

Schraffierte Fläche stellt die mittlere Nettobilanz dar mit individuell abgeschätzter Fehlergrenze. Schraffierte und weiße Fläche nach oben ergibt Winterbilanz, nach unten Sommerbilanz. Gestrichelte Linie ergibt Nettogewinn in Form von «superimposed ice». Tabelliert ist der numerische Wert der mitteleren spezifischen Nettobilanz.

Dies wird in erster Linie auf die dämpfende Wirkung des hoch gelegenen Akkumulationsgebietes des White Glacier zurückgeführt, in dem kaum je Ablation, dafür aber gleich bleibende Winterakkumulation herrscht. Eine ebenso gute Korrelation ergibt sich mit den Massenbilanzen der Meighen Ice Cap ( $r=0.97$ bei $n=7)$ auf Meighen Island, $160 \mathrm{~km}$ nordwestlich des Baby Glacier.
Gerade noch signifikant ist sie (auf dem 5\% Niveau) für den Nordwestsektor der Devon Ice Cap auf Devon Island $(\mathrm{r}=0.64$ bei $\mathrm{n}=9), 480 \mathrm{~km}$ südöstlich. Mit dem Decade Glacier, $1160 \mathrm{~km}$ südöstlich auf Baffin Island, kann keine signifikante Korrelation mehr gefunden werden (Quellen für Nettobilanzen der Gletscher außerhalb Axel Heiberg Island: KOERNER, 1970; PATERSON, 1969; IAHS, 1972). 
Die weiträumige Korrelation der Nettobilanzen der verschiedenen Gletscher ist durch die enge Verknüpfung mit den Sommertemperaturen zu erklären, die ihrerseits über weite Gebiete ähnlich sind. So ist auch die Nettobilanz des Baby Glacier fast gleich gut mit den Sommertemperaturen von Isachsen, $180 \mathrm{~km}$ westlich des Expeditionsgebietes, korreliert $(r=0.92$ bei $\mathrm{n}=15)$ wie mit denen des Basislagers $(\mathrm{r}=0.93$ bei $\mathrm{n}=10$ ). Der Zusammenhang mit dem ziemlich nahe gelegenen Eureka (110 km nordöstlich) ist bedeutend schlechter $(r=0.74$ bei $n=15)$. Die Station Eureka ist allerdings anormal warm, zudem scheinen Windströmungen und Gebirgszüge einen Einfluß zu haben. Eine verständliche Verknüpfung zwischen den Winterbilanzen des Gletschers und den Niederschlägen an den Stationen konnte nicht gefunden werden, unter anderem deshalb, weil schon die Niederschläge an den Stationen schlechter miteinander korreliert sind als die Temperaturen.

Die starke Abhängigkeit der Nettobilanz des Baby Glacier vom Temperaturregime der Umgebung, die im Vergleich zum White Glacier größeren Bilanzschwankungen sowie der relativ kleine Aufwand an Feldarbeit machen den Gletscher zu einem interessanten Studienobjekt der Wechselbeziehungen der Klima-Gletscher. So zeigen die Regressionsrechnungen der Nettobilanz als Funktion der Sommertemperaturen von Isachsen oder des Basislagers, daß pro $1^{\circ} \mathrm{C}$ niedrigerer Sommertemperatur die Nettobilanz um $30 \mathrm{~cm}$ WE steigt; der Gletscher reagiert also meßbar auf Temperaturschwankungen von Bruchteilen eines Grades!

Diese Untersuchung war nur möglich dank der jahrelangen Vorarbeit, die von der Axel Heiberg Island Expedition geleistet wurde. Speziell sei die Mithilfe von Koni Steffen und Jakob Weiß bei der Feldarbeit verdankt. Atsumu Ohmura und Roger Braithwaite haben bei der Auswertung wertvolle Ratschläge erteilt. Finanzielle Unterstützung für die Studie wurde vom
National Research Council of Canada (durch Grant No. A-2662 an Dr. F. Müller) und dem Geographischen Institut der ETH Zürich gewährt. Das Polar Continental Shelf Project der kanadischen Regierung stellte die logistischen Mittel zur Verfügung.

\section{Literatur}

ADAMS, W. P. 1966. Ablation and Runoff on the White Glacier, Axel Heiberg Island. Axel Heiberg Island Research Report, McGill University, Montreal.

BAIRD, P. D. 1952. Method of the nourishment of the Barnes Ice Cap. Journal of Glaciology, Vol. 2, No. 11, p. 2-9.

IAHS, Unesco. 1973. Fluctuations of Glaciers. International Commission on Snow and Ice of the International Association of Hydrological Sciences, Paris.

KOERNER, F. 1970. The mass balance of the Devon Ice Cap, N.W.T., Canada. Journal of Glaciology, Vol.9, No. 57, p. 325-336.

MÜLLER, F. et al. 1961. Axel Heiberg Island, Preliminary Report 1959-1960. McGill University, Montreal.

MÚLLER, F. 1962. Glacier mass budget studies on Axel Heiberg Island, Canadian Arctic Archipelago. Symposium of Obergurgl, International Association of Scientific Hydrology, Publ. No. 58, p. 131-142.

MÜLLER, F. 1962. Zonation in the accumulation area of the glaciers of Axal Heiberg Island, Northwest Territories, Canada. Journal of Glaciology, Vol. 4, No. 33, p. 302-310.

MÜLLER, F. et al. 1963. Preliminary Report 1961-1962. Axel Heiberg Island Research Reports, McGill University, Montreal.

PATERSON, w. S. P. 1969. The Meighen Ice Cap, Arctic Canada: accumulation, ablation and flow. Journal of Glaciology, Vol. 8, No. 54, p. 341-352. 
Abb. 1 Gletschergestauter Astro Lake mit Eisbergen, im Vordergrund Thombson Glacier, Axel Heiberg Island, N.W.T. (Juli 1962). Foto: H. Maag

Abb. 2 Basislager am Colour Lake mit einjährigem Seeeis. Im Hintergrund Schwemmebene des Expedition River (Juli 1962). Foto: F. Müller
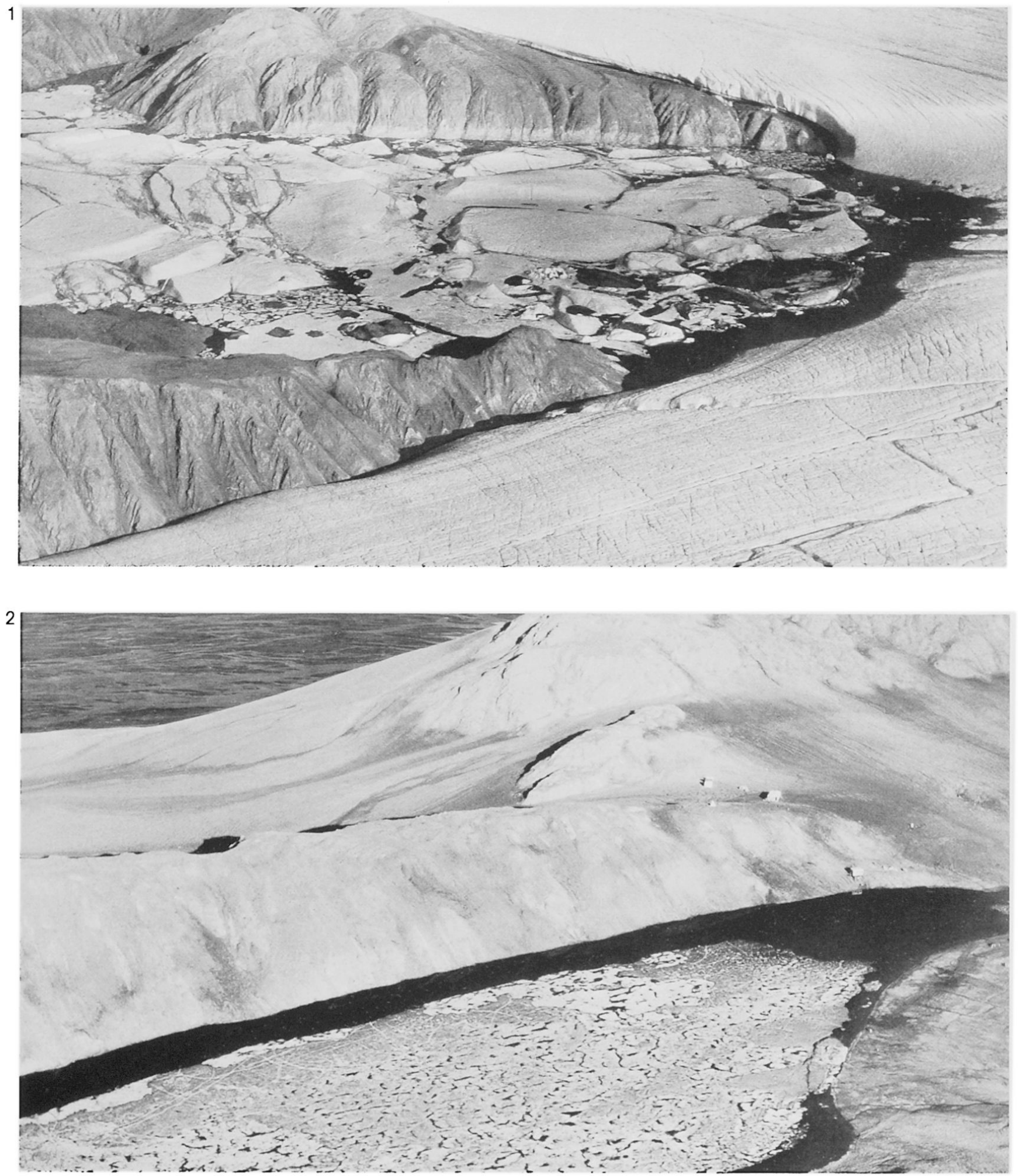